\title{
FINITE ONE-LOOP RADIATIVE CORRECTIONS IN THE LORENTZ- AND CPT-VIOLATING QED EXTENSION
}

\author{
D.H.T. FRANCO and A.H. GOMES* \\ Universidade Federal de Viçosa, Viçosa, 36570-000, Minas Gerais, Brazil \\ ${ }^{*}$ E-mail: andre.herkenhoff@ufv.br
}

We report on our current progress in the study of finite one-loop radiative corrections in the minimal Lorentz- and CPT-violating QED extension.

\section{Introduction}

Quantum gravity effects coming from Planck scale energies may appear as small violations of fundamental laws in the limit of low energies. One possibility is the breaking of both Lorentz and CPT symmetries. A comprehensive investigation of this case is made viable, for instance, in the framework known as the Standard Model Extension, ${ }^{1}$ the most general effective model comprising particle Lorentz and CPT violation but coordinate invariant. The minimal extension respects the Standard Model $S U(3) \otimes S U(2) \otimes U(1)$ gauge symmetry and is power-counting renormalizable. In this proceedings contribution we report our progress in the study of finite one-loop radiative corrections in the minimal Lorentz- and CPT-violating QED extension.

\section{The QED Extension and setup for one-loop evaluations}

The Lorentz- and CPT-violating QED extension for one Dirac fermion can be described by the Lagrange density ${ }^{1}$

$$
\begin{aligned}
\mathcal{L}= & i \bar{\psi}\left(\gamma^{\mu}+\Gamma_{1}^{\mu}\right) D_{\mu} \psi-\bar{\psi}\left(m+M_{1}\right) \psi-\frac{1}{4} F^{\mu \nu} F_{\mu \nu} \\
& -\frac{1}{4}\left(k_{F}\right)_{\mu \nu \rho \sigma} F^{\mu \nu} F^{\rho \sigma}+\frac{1}{2}\left(k_{A F}\right)^{\mu} \epsilon_{\mu \nu \rho \sigma} A^{\nu} F^{\rho \sigma},
\end{aligned}
$$

where Lorentz violation (LV) is parameterized by coefficients in the form of constant background fields - see the last two terms of Eq. (1) and the 
definitions

$$
\begin{gathered}
\Gamma_{1}^{\mu} \equiv c^{\lambda \mu} \gamma_{\lambda}+d^{\lambda \mu} \gamma_{5} \gamma_{\lambda}+e^{\mu}+i f^{\mu} \gamma_{5}+\frac{1}{2} g^{\kappa \lambda \mu} \sigma_{\kappa \lambda}, \\
M_{1} \equiv a^{\mu} \gamma_{\mu}+b^{\mu} \gamma_{5} \gamma_{\mu}+\frac{1}{2} H^{\mu \nu} \sigma_{\mu \nu} .
\end{gathered}
$$

We also add to (1) a photon mass term $\frac{1}{2} \lambda^{2} A^{2}$ to control infrared divergences and a gauge-fixing piece $-\frac{1}{2 \alpha}\left(\partial_{\mu} A^{\mu}\right)^{2}$.

We follow the guidelines presented in Ref. 2 to deal with one-loop radiative corrections: evaluations are performed in the so-called concordant frames to avoid issues with the perturbative expansion; nonlinear LV is disregarded because it could lead to effects comparable to multiloop ones, which we do not consider; and LV pieces of the Lagrange density (1) are treated as new interaction vertices, effectively entering as propagator or vertex insertions, as allowed by the experimental smallness of LV.

\section{Results and discussion}

As a first step, we considered only the $b_{\mu}$ and $\left(k_{A F}\right)_{\mu}$ coefficients. Results for the photon self-energy can be found in Ref. 1, so our goal was first the vertex correction diagram, Fig. 1(a). The electron self-energy was used so far only to check the related gauge identity (more details below), and its evaluation will be our next goal. The LV contribution with one $b_{\mu}$ insertion comes from two diagrams, Figs. 1(b) and 1(c), with the new Feynman rule

$$
\longrightarrow \times=-i b_{\mu} \gamma_{5} \gamma^{\mu},
$$

and the contribution from $\left(k_{A F}\right)_{\mu}$ comes from the one in Fig. 1(d), where

$$
\mu ح \propto \nu=2\left(k_{A F}\right)^{\alpha} \varepsilon_{\alpha \mu \beta \nu} k^{\beta} .
$$

These insertions lower the superficial degree of divergence of vertexcorrection loop integrals, making them actually finite, avoiding complications related to $\gamma_{5}$.

Aiming for generality, we computed both cases with fermions and photon off shell, i.e., $P^{2} \neq m^{2} \neq p^{2}$ and $q^{2} \neq 0$, and we made no use of the Dirac equation. Since Gordon identities are unavailable to make further simplifications - they are valid only on shell - the final result itself is not very illuminating because of its size and structure, but it is worth mentioning that because we do not use $P^{2}=m^{2}=p^{2}$ our expressions contain not only pieces symmetric under $P \leftrightarrow p$ but also pieces that are antisymmetric. On the other hand, symmetry under $P \leftrightarrow p$ is recovered when the fermions go 


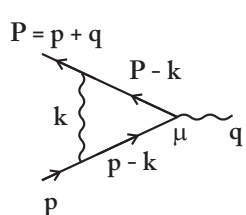

(a)

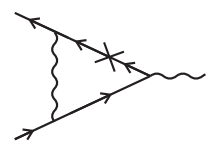

(b)

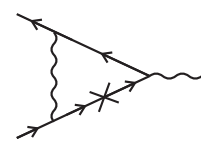

(c)

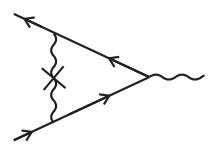

(d)

Fig. 1. (a) Conventional QED vertex correction and (b)-(d) Lorentz-violating insertions.

on shell, and the final expressions are greatly simplified. For instance, the $\mathrm{LV}$ correction due to $\left(k_{A F}\right)_{\mu}$ has the structural form

$$
\begin{aligned}
\Gamma_{k_{A F}}^{\mu} \sim & \left\{\not k_{A F} \gamma_{5}(P+p)^{\mu}, \gamma_{5} \sigma^{\mu \nu}(P+p)_{\nu} k_{A F} \cdot(P+p),\right. \\
& \left.\gamma^{\mu} \gamma_{5} k_{A F} \cdot(P+p), \gamma_{5} \sigma^{\kappa \lambda}\left(k_{A F}\right)_{\kappa}(P+p)_{\lambda}(P+p)^{\mu}\right\},
\end{aligned}
$$

and the result from the $b^{\mu}$ insertion has the same structure but with the additional form $\gamma_{5} \sigma^{\mu \nu} b_{\nu}$, which vanishes for the $\left(k_{A F}\right)_{\mu}$ contribution.

As a consistency check, we verified the Ward-Takashi identity, $q_{\mu} \Gamma^{\mu}=$ $\Sigma(p+q)-\Sigma(p)$, for both cases up to order $q^{2}$ with arbitrary IR regulator $\lambda$, but so far with fermions on shell - the off shell case is still to be done. The Ward-Takashi identity demands the evaluation of LV contributions to the electron self energy, which are divergent. Nevertheless, only the finite piece of this integral is momentum dependent and contributes to the right-hand side of the Ward-Takashi identity. It is interesting to notice that, different from the conventional QED case, $q_{\mu} \Gamma^{\mu} \neq 0$ even when the Dirac equation is used for the external leg spinors: even in this situation the momentum shift in the electron self energy still contributes to the identity.

The next step is to establish the finite contributions coming also from the divergent piece of the electron self energy, which may require a careful choice of subtraction points. Applications in physical processes will also be envisaged, as well as the analysis for other LV coefficients.

\section{Acknowledgments}

Financial support was provided by CNPq (Brazil).

\section{References}

1. D. Colladay, and V.A. Kostelecký, Phys. Rev. D 58, 116002 (1998).

2. V.A. Kostelecký, C.D. Lane, and A.G.M. Pickering, Phys. Rev. D 65, 056006 (2002). 